\title{
Yowie: Information Extraction in a Service Enabled World
}

\author{
Marek Kowalkiewicz and Konrad Jünemann \\ SAP Research, 133 Mary Street, Brisbane, Australia \\ \{marek.kowalkiewicz, konrad.juenemann\} @sap.com
}

\begin{abstract}
Service Oriented Computing is a potential enabler for popular applications of Named Entity Recognition and Information Extraction. In this demo we show an example of such an application and discuss how Service Oriented Architecture (SOA) makes the application fully flexible and easily extensible. The application brings SOA close to the end-user and gives possibilities hardly possible with other approaches.
\end{abstract}

Keywords: SOA applications, natural language processing.

\section{Introduction}

The domain of Natural Language Processing (NLP) has reached a state of maturity where commercial applications are possible and reliability of such applications becomes acceptable. Although some of the areas of NLP, for instance Information Extraction (IE) or Named Entity Recognition (NER), are particularly advanced [1|2], there still is no popular "killer application" that can demonstrate high potential of NLP. That holds for both enterprise and non-enterprise world.

There are some examples of NER applied in popular applications. However, these systems are not extensible by end-users in any way, and there is no possibility to (a) enable recognition of new types of entities or (b) provide users with other, alternative actions that can be performed with the recognized entities. As there are more and more information processing services available both in Internet and in enterprise software, SOA can provide a solution to this problem.

Ability to add new types of entity recognition on top of standard ones is especially important in enterprise context. Date, place name, or human name recognition, often provided by NLP systems, can be extended with features such as product name (code), customer name or contract number recognition, providing much more automation to the enterprise world. Ability to offer new services that process entities brings benefits to both enterprise and non-enterprise worlds.

Since relevance of actions and services, together with their rankings, varies based on numerous factors, high flexibility and ability to add and replace system components become useful. In this demonstration we showcase Yowie, a NER system built following SOA guidelines. Thanks to SOA, Yowie is fully extensible and can make use of external services.

A. Bouguettaya, I. Krueger, and T. Margaria (Eds.): ICSOC 2008, LNCS 5364, pp. 732 733, 2008.

(C) Springer-Verlag Berlin Heidelberg 2008 


\section{Description of the System}

Yowie provides a link between business productivity software and external services local desktop, enterprise systems and Internet vendors. The link that Yowie provides is based on the assumption that certain fragments of text documents contain enough information to create automatic links to data objects and services available in external systems.

Yowie consists of four main components: (1) a set of plugins, responsible for accessing local applications and communicating with Yowie, (2) a core, acting as a mediator between all other components, (3) a set of extractors, recognizing entities in documents, and (4) a set of service wrappers providing access to services and information related to extracted entities. The components are loosely coupled and each of them can be easily replaced. The main novel characteristics of Yowie include:

Integration of NER and IE services. Yowie can integrate various NER and IE services in one application following the SOA paradigm. Other existing approaches are either hard coded or do not integrate various services at all;

NER and IE: entry points for service consumption. NER and IE can be used for service selection. Yowie is an example of an application that can consume services offered by a service broker and choose the relevant ones;

A potential for an SOA-enabled killer application. By integrating NER, IE, and SOA in end-user applications, Yowie has a potential of becoming popular and being a visible example of SOA in end-user applications.

\section{Functions and Features to be Demonstrated}

During the demo we will showcase functionality of Yowie. During the presentation we will focus on one Yowie plugin, Yowie for MS Outlook. We are going to demonstrate the following.

- Ability to recognize various types of entities in processed documents using a simple email as an example.

- Ability to provide end users with related services (local, Web, and enterprise services). For each of the recognized entities we will show information and service access provided by Yowie.

- Ability to add or replace recognizers for new types of named entities. Yowie can be easily extended by adding new services that recognize named entities.

- Ability to add or replace offered services based on recognized entities. New services that use recognized entities can be easily added to the system.

\section{References}

1. Maynard, D., Tablan, V., Ursu, C., Cunningham, H., Wilks, Y.: Named Entity Recognition from Diverse Text Types. Recent Advances in NLP (2001)

2. Nahm, U., Mooney, R.: Text Mining with IE. In: Proc. of the AAAI 2002 Spring Symposium on Mining Answers from Texts and Knowledge Bases (2002) 